\title{
Fatores de risco associados à ocorrência de linfadenite em suínos na fase de crescimento-terminação ${ }^{1}$
}

\author{
Armando Lopes Amaral ${ }^{2}$, Nelson Morés ${ }^{3}$, Waldomiro Barioni Júnior ${ }^{4}$, Lauren \\ Ventura ${ }^{5}$, Roberto Aguilar Machado da Silva ${ }^{6}$ e Virgínia Santiago da Silva ${ }^{2}$
}

\begin{abstract}
Amaral A.L., Morés N., Barioni W.]., Ventura L., Silva R.A.M. \& Silva V.S. 2004. [Risk factors at the growth-termination phase associated with the occurrence of lymphadenitis in swine.] Fatores de risco associados à ocorrência de linfadenite em suínos na fase de crescimento-terminação. Pesquisa Veterinária Brasileira 24(3):120-122. Depto Epidemiologia, Embrapa Suínos e Aves, Cx. Postal 21, Concórdia, SC 89700-000, Brazil. E-mail: armando@ cnpsa.embrapa.br

An epidemiological study in 60 units of swine termination (UT), pertaining to producers integrated with the main Agribusiness of the States of Santa Catarina and Rio Grande do Sul. These units had been selected due to the occurrence of lymphadenitis in slaughtered pigs. The batch size studied in each UT varied from 22 to 960 pigs. Theanimals were considered positive or negative for lymphadenitis, following the evaluation routine of the Service of Federal Inspection (SIF) at slaughter. For data collection at the UTs, a questionnaire with 156 variables was applied at a single visit to the farms. In the 60 UT studied, the percentage of positive animals for lymphadenitis was $10.37 \%$ Among the variables analyzed, nine risk factors were identified (explicative variables) and associated with the occurrence of lymphadenitis (objective variables). The explicative variables were: bad hygiene of the feeders and drinking fountains, untreated drinking water, bad conservation of the facilities, use of the same truck for ration and animal transportation, production of ration inside the farm, free access of other animals to the ingredients of ration, storage of ready usable ration in boxes or bags and handling of the production of animals in an continuous system.
\end{abstract}

INDEX TERMS: Swine, risk factors, lymphadenitis.

RESUM O.- Realizou-se estudo epidemiológico em 60 unidades de terminação de suínos (UT), pertencentes a produtores integrados às principais agroindústrias dos Estados de Santa Catarina e Rio Grande do Sul. Esses produtores foram selecionados em função da ocorrência de linfadenite em lotes de suínos abatidos. 0 tamanho dos lotes acompanhados em cada UT variou de 22 a 960 suínos. Os animais foram considerados positivos ou negativos para linfadenite, pela avaliação de rotina do Serviço de Inspeção Federal (SIF) no abate. Para coleta dos dados nas UT, foi aplicado um questionário com 156 variáveis em umaúnica visita às granjas. Nos lotes das 60 UT estudadas, a percentagem de

${ }^{1}$ Recebido em 14 de janeiro de 2003.

Aceito para publicação em 23 outubro de 2003.

2 Embrapa Suínos e Aves, Depto Epidemiologia , Cx. Postal 21, Concórdia, SC 89700-000. E-mail: armando@ cnpsa.embrapa.br

${ }^{3}$ Embrapa Suínos e Aves, Depto Patologia Animal.

${ }^{4}$ Embrapa Suínos e Aves, Depto Estatística.

${ }^{5}$ Convênio ACCS, AINCADESC e Embrapa Suínos e Aves, Depto Sanidade Suína.

${ }^{6}$ Embrapa Pantanal, Depto Patologia Clínica, Cx. Postal 109, Corumbá, MS 79320-900. animais positivos para linfadenite foi de $10,37 \%$ Do conjunto de variáveis analisadas foram identificados nove fatores de risco (variáveis explicativas) associados à ocorrência de linfadenite (variável objetiva), os quais são: a má higiene dos comedouros e bebedouros, a água não tratada, a má conservação das instalações, o transporte de ração e animais com o mesmo caminhão, a produção de ração na propriedade, 0 acesso de outros animais à fabrica de ração, a estocagem de ração pronta em caixas ou sacos e o manejo na produção de animais em sistema contínuo.

TERMOS DE INDEXAÇÃO: Suínos, fatores de risco, linfadenite.

\section{INTRODUÇÃO}

A linfadenite granulomatosa nos suínos éum tipo de lesão detectada durante a inspeção de rotina nos abatedouros, cujo impacto econômico para a região sul do Brasil, devido a condenação de vísceras e carcaças com destino condicionado, tem sido estimado em 8 milhões de reais ao ano (Martins et al. 2001). As micobactérias do complexo Mycobacterium avium (MAC) são os agentes principais destas lesões na região Sul do Brasil (Morés et al. 1999). A doença apresenta evolução crônica, podendo levar mais de 4 meses desde a infecção até o aparecimento de lesões visíveis macroscopicamente, tornando difícil o rastreamento da 
fonte de infecção e, conseqüentemente, o controle da enfermidade na granja (Carpenter\& Hird 1986). Em função da importância econômica na cadeia produtiva e do seu potencial zoonótico, faz-se necessário um melhor entendimento da epidemiologia da doença para a prevenção e diminuição da prevalência nas granjas de suínos nesta região. Poucos são os trabalhos realizados nessa direção (Oliveira 1989, Silva 1998), o que tem dificultado a implementação de estratégias para controle. 0 objetivo deste trabalho é identificar o conjunto de fatores de risco na fase de crescimento-terminação que melhor explicam a ocorrência de linfadenites em criaçõ̃es de suínos da região sul do Brasil.

\section{MATERIAL E MÉTODOS}

Realizou-se estudo epidemiológico durante o período de abril de 1999 a setembro de 2000 em 60 unidades de terminação de suínos (UT), integrados às principais agroindústrias, situadas em Santa Catarina e Rio Grande do Sul. Estes produtores foram selecionados em função da prevalência de animais com lesões de linfadenite granulomatosa em lotes de suínos abatidos. 0 tamanho dos lotes acompanhados em cada UT variou de 22 a 960 suínos. Os animais foram considerados positivos ou negativos para linfadenite pela avaliação de rotina do Serviço de Inspeção Federal no abate. Para coleta dos dados nas UT foi elaborado um questionário com informações da literatura sobre possíveis fatores de risco para a doença estudada. 0 questionário continha 156 variáveis, incluindo variáveis quantitativas (contínuas) e qualitativas (categóricas) que caracterizam o ecossistema do crescimento e terminação, preenchido em uma única visita às granjas.

Na análise estatística dos dados foram aplicados os procedimentos sugeridos por Madec \& Josse (1984), para estudos ecopatológicos, com o objetivo de analisar em conjunto um grande número de variáveis. Inicialmente, os dados foram submetidos a uma análise exploratória univariada, através do teste do $c^{2}$. As variáveis explicativas (possíveis fatores de risco) que apresentaram $\mathrm{pf} 0,20$ no teste $c^{2} \mathrm{com}$ a variável objetiva (\% de linfadenite no lote) foram submetidas a análise multivariada e análise de correspondência múltipla (ACM). Esta última teve como objetivo estudar as relações e semelhanças existentes entre linhas e colunas de uma tabela de contingência e representá-las graficamente, proporcionando uma interpretação simples dos resultados. Os softwares estatísticos adotados para análise dos dados foram: Statistical Analysis System (SAS 1996) e Sistema Portátil de Análise de Dados Numéricos (Centre 1994).

\section{RESULTADOS E DISCUSSÃO}

Das 156 variáveis estudadas, foram selecionadas 15 para serem submetidas aos testes estatísticos multivariados. No Quadro 1 está a variável objetiva (porcentagem de animais com linfadenite no lote) subdividida em quatro classes em função da prevalência de animais com linfadenite. No Quadro 2 estão os fatores de risco (variáveis explicativas) associados a ocorrência de linfadenite no lote acompanhado na granja.

Dos lotes acompanhados nas 60 UT, a porcentagem média de animais positivos para linfadenites foi de $10,37 \%$ Sobre o conjunto de variáveis analisadas foram identificados nove fatores de risco (variáveis explicativas) associados a ocorrência de linfadenite (variável objetiva). A Fig. 1 apresenta o mapa dos fatores de risco associados às diferentes categorias de ocorrência de linfadenite (LINF1, LINF2, LINF3 e LINF4). Nesse mapa, observa-se que a má qualidade da higiene dos comedouros e bebedouros ( $\mathrm{HICl}, \mathrm{HIB1}$ ), a água não tratada (AGUA1), a má conservação das instalações (INST1), o transporte de ração e animais com o mesmo caminhão (TRI1), a produção de ração na propriedade (RAC1), 0 acesso de outros animaisà

Quadro 1. Descrição da variável objetiva (porcentagem de animais com linfadenite) nos lotes acompanhados com as classes, freqüências absolutas (Abs) e relativas $(\%$, e a prevalência de linfadenite

\begin{tabular}{llccc}
\hline \multicolumn{1}{c}{ Descrição } & \multicolumn{1}{c}{ Classes } & \multicolumn{2}{c}{ Freqüências } & Linfadenite \\
\cline { 3 - 4 } & & Abs. & $\%$ & $\%$ \\
\hline Prevalência de lin- & LINFA1: $\leq 1,0$ & 12 & 20 & 0,19 \\
fadenite no lote & LINFA2: $>1,0 \leq 5,0$ & 15 & 25 & 2,82 \\
& LINFA3: $>5,0 \leq 10,0$ & 18 & 30 & 7,39 \\
& LINFA4: $>10,0$ & 15 & 25 & 29,66
\end{tabular}

Quadro 2. Descrição das variáveis explicativas (fatores de risco) associadas à prevalência de linfadenite com as classes e as freqüências absolutas (Abs) e relativas (\%)

\begin{tabular}{|c|c|c|c|c|}
\hline \multirow[t]{2}{*}{ Descrição } & \multirow[t]{2}{*}{ Classes $^{a}$} & \multicolumn{2}{|c|}{ Freqüências } & \multirow{2}{*}{$\begin{array}{c}\text { Linfadenite } \\
\%\end{array}$} \\
\hline & & Abs. & $\%$ & \\
\hline \multirow{2}{*}{$\begin{array}{l}\text { 1. Caminhão que transporta insumos e/ou } \\
\text { rações, também, transporta animais }\end{array}$} & $\underline{T R A} \underline{\underline{a}}=\operatorname{sim}$ & 15 & 25 & 18,77 \\
\hline & $\overline{\text { TRA2 }} \overline{=\text { não }}$ & 45 & 75 & 7,58 \\
\hline \multirow[t]{2}{*}{ 2. Produz ração na propriedade } & $\underline{\mathrm{RACl}}=\operatorname{sim}$ & 15 & 25 & 7,86 \\
\hline & $\overline{\mathrm{RAC2}}=$ não & 45 & 75 & 17,92 \\
\hline \multirow{2}{*}{$\begin{array}{l}\text { 3. Acesso de animais à fabrica de ração } \\
\text { (gatos, galinhas, cães, ...) }\end{array}$} & $\underline{A F A 1}=\operatorname{sim}$ & 15 & 25 & 18,61 \\
\hline & $\overline{\mathrm{AFA} 2}=$ não & 45 & 75 & 7,63 \\
\hline \multirow[t]{2}{*}{ 4. Forma de estocagem da ração pronta } & $\underline{\text { REST1 } 1 \text { = caixas abertas ou sacos }}$ & 15 & 25 & 19,03 \\
\hline & $\overline{\text { REST2 }=\text { silos }}$ & 45 & 75 & 7,49 \\
\hline \multirow[t]{2}{*}{ 5. Tratamento da água fornecida aos suínos } & $\underline{\text { AGUA1 }=\text { não tratada }}$ & 45 & 75 & 12,98 \\
\hline & AGUA2 = tratada & 15 & 25 & 2,56 \\
\hline \multirow[t]{2}{*}{ 6. Manejo da instalação } & $\underline{V A Z 1}=$ contínuo & 11 & 18 & 20,76 \\
\hline & 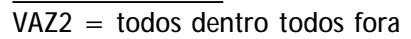 & 49 & 82 & 8,04 \\
\hline \multirow[t]{2}{*}{ 7. Higiene dos comedouros por ocasião da visita } & $\underline{\mathrm{HICl}}=$ sujo & 43 & 71 & 19,65 \\
\hline & $\overline{\mathrm{HIC2}}=\operatorname{limp} 0$ & 17 & 29 & 6,71 \\
\hline \multirow[t]{2}{*}{ 8. Higiene dos bebedouros por ocasião da visita } & $\underline{\mathrm{HIB1}}=$ sujo & 14 & 24 & 10,51 \\
\hline & $\overline{\mathrm{HIB} 2}=$ limpo & 46 & 76 & 10,33 \\
\hline \multirow{2}{*}{$\begin{array}{l}\text { 9. Estado de conservação das instalações } \\
\text { fatores de risco para linfadenite. }\end{array}$} & $\underline{\mathrm{INST} 1}=$ regular ou ruim & 24 & 40 & \\
\hline & $\overline{\text { INST2 }=\text { bom }}$ & 36 & 60 & 9,13 \\
\hline
\end{tabular}

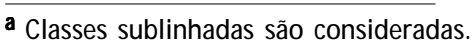




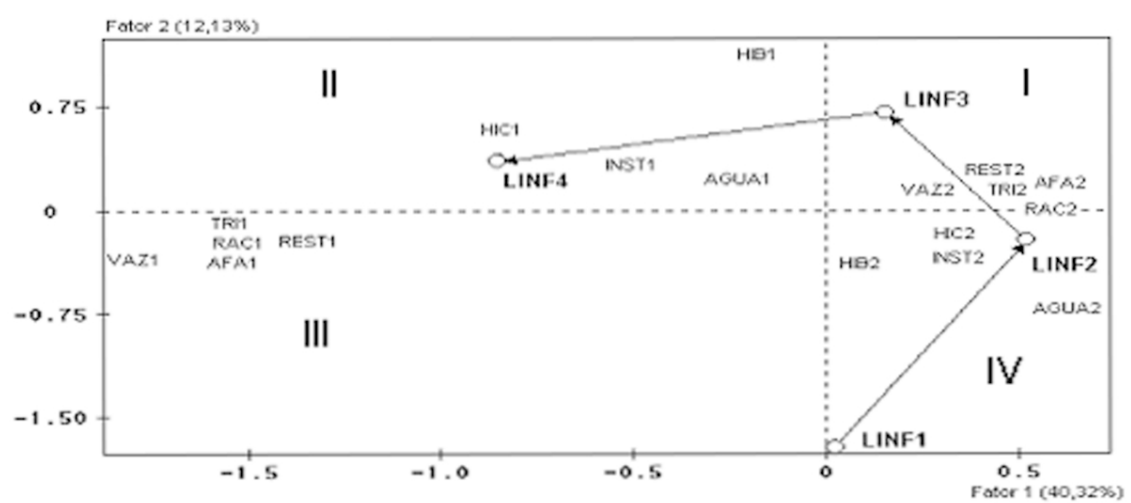

Fig. 1. Mapa dos fatores de risco associados à linfadenite, gerado pela análise de correspondência múltipla.

fabrica de ração (AFA1), a estocagem de ração pronta em caixas abertas ou sacos (REST1) e o manejo na produção dos suínos em sistema contínuo (VAZ1) são categorias dos fatores de risco associados a alta prevalência de linfadenite (LINF3 e LINF4) que estão nos quadrantes I, II e III do mapa. Por outro lado, observa-se também que os comedouros e bebedouros limpos (HIC2, HIB2), o bom estado de conservação das instalações (INST2), a água tratada (AGUA2), o caminhão que só transporta ração (TRI2), a compra de ração pronta (RAC2), não ter acesso de animais na fábrica de ração (AFA2), estocagem da ração pronta em silos (REST2) e o manejo na produção dos suínos em lotes com vazio sanitário (VAZ2) são categorias de fatores associados à ausência e baixa prevalência de linfadenite (LINF1 e LINF2) que estão no quadranteIV do mapa.

Em outro estudo epidemiológico do tipo caso-controle, nessa mesma região (Silva et al. 2001), em sistemas de produção de suínos em ciclo completo, foram identificados três fatores de risco associados a ocorrência de linfadenite, sendo dois ligados às condições higiênicas na creche e um ao tamanho da criação. Dos nove fatores identificados nesse estudo, seis (TRA1, REST1, AGUA1, VAZ1, HICl e HIB1) também estão ligados a questões higiênicas e outros dois fatores (RACl, AFA1) estão ligados às questões de biossegurança do alimento fornecido aos suínos. $A$ presença de aves domésticas e ou silvestres nas instalações já havia sido relatado como fator de risco para linfadenite em suínos (Alfredsen \& Skjerve 1993). A maior ocorrência de linfadenite, associada à má qualidade da higiene nos rebanhos, deve-se à maior possibilidade dos suínos se infectarem por MAC, uma vez que esta bactéria é eliminada nas fezes de suínos portadores (Cole et al. 1980, Silva 1998), e a principal via de infecção é a oral, tendo como origem material contaminado: alimentos, cama, água, solo e fezes do próprio suíno (Oliveira 1989).

Alguns autores têm relacionado o uso da cama sobreposta na suinocultura com ocorrência de lesões de linfadenite (Brooks 1971, Windsor et al. 1984). Charette et al. (1988) verificaram que uma amostra de M ycobacterium avium, foi capaz de sobreviver durante um ano acondicionada em sacos plásticos na maravalha em temperatura variando de 20 a $30^{\circ} \mathrm{C}$. No presente estudo, as UT acompanhadas não utilizaram qual quer tipo de cama.

Desta forma, foram identificados nove fatores de risco associados à ocorrência de linfadenite em crescimento e terminação de suínos em piso compacto. Todos os fatores de risco estiveram relacionados com a limpeza e desinfecção das instalações, equipamentos e a qualidade higiênica dos alimentos fornecidos aos animais.

\section{REFERÊNCIAS}

Alfredsen S. \& Skjerve E. 1993. An abattoir-based case-control study of risk factors for mycobacteriosis in Norwegian swine. Prev. Vet. Med. 15:253259.

Brooks O.H. 1971. Observations on outbreaks of Battey type mycobacteriosis in pigs raised on deep litter. Aust. Vet. J. 47:424-427.

Carpenter T.E. \& Hird D.W. 1986. Time series analysis of mycobacterioses in California slaughter. Prev. Vet. Med. 3:559-572.

Centre Internacional de Statistique et D'informatique Appliquées (SaintMandé, France) 1994. Oferta estudante SPADN integrado: version P.C. Saint-Mandé. 215p.

Charette R., Martineau G.P., Turcatte C. \& Higgins R. 1988. Epidemiological aspects of mycobacterial lymphadenitis in swine: a case report. Proc. 10th Congr. Int. Pig Vet. Society, 156.

Cole J.R., Sangster L.T., Herron D. \& Pulrsell A.R. 1980. Swine mycobacterial infeccion: epidemiology, pathogenesis and prevention, p. 198. In: N.C. Nielson, P. Hogh \& N. Bile (ed.) Proc. IPVS Congress, Copenhagen. International Pig Veterinary Society, Cambridge, UK.

Madec F. \& Josse J. 1984. Utilization des methodes d'analyse des donnes pour l'etude de maladies d'elevage application du porc. Epid. Sante Anim. 6:35-63.

Martins L.S. 2001. Epidemiologia e controle das micobacterioses em suínos no Sul do Brasil: estimativa do impacto econômico e estudo da sazonalidade. Dissertação (Mestrado), FMVZ, Universidade de São Paulo, São Paulo, 51 p.

Morés N., Dutra V., Silva V.S., Pereira M.A.C., Yamamoto T.M., Ventura L., Barioni Jr W., Piffer I.A., Vidal C.E.S., Silva R.A.M.S., Oliveira S.R., Kramer B., Ferreira Neto J.S., Balian S.C. \& Leão S.C. 1999. Linfadenite granulomatosa em suínos da região Sul do Brasil: principais linfonodos afetados, destino das carcaças e agentes envolvidos. IX Congr. Bras. Vet. Especialistas em Suínos, Belo Horizonte, MG, p. 223-224.

Oliveira S. J. 1989. Tuberculose em suínos. Hora Vet., Porto Alegre, 49:3136.

SAS Institute Inc. SAS System for microsoft windows: release 6.12, Cary, NC, USA, 1996. 1 CD- ROM.

Silva V.S. 1998. Estudo da transmissão horizontal de Mycobacterium aviumintracellulare em suínos. Dissertação (Mestrado), Universidade Federal de Pelotas, Pelotas, RS, $47 \mathrm{p}$.

Silva V.S., Morés N., Ferreira F., Silva M.F., Dias R.A., Balian S., Dutra V., Leão S.C., Pinheiro S.R., Sakamoto S.M. \& Ferreira Neto J.S. 2001. Identificação dos fatores de risco associados à ocorrência de micobacterioses no Sul do Brasil. In: Conferência Virtual sobre Saúde de Suínos, Concórdia, SC. Anais eletrónicos... Embrapa Suínos e Aves, Concórdia. Disponível em :<http//www.cnpsa.embrapa.br> acesso em: 19 maio 2001.

Windsor R.S., Durrant D.S. \& Burn K.J. 1984. Avian tuberculosis in pigs: Mycobacterium intracellulare infection in a breeding herd. Vet. Rec. 114:497500 . 